\title{
Nondestructive Measurement of Sugar Content in Navel Orange Based on Vis-NIR Spectroscopy
}

\author{
Chunsheng Luo ${ }^{1}$, Long Xue ${ }^{2}$, Muhua Liu ${ }^{1, *}$, Jing $\mathrm{Li}^{1}$, and Xiao Wang ${ }^{1}$ \\ ${ }^{1}$ Engineering College, Jiangxi Agricultural University, Nanchang, Jiangxi 330045, P. R. China \\ ${ }^{2}$ School of Mechanical and Electronical Engineering, East China JiaoTong University, \\ Nanchang, Jiangxi 330013, P.R. China
}

\begin{abstract}
The Vis-NIR spectroscopy technology was studied on nondestructive measurement of sugar content in navel orange. Three different spectral ranges of $450-1000 \mathrm{~nm}, 1000-1800 \mathrm{~nm}$ and $450-1800 \mathrm{~nm}$ were selected, respectively and five different spectral pre-processing methods of Standard Normal Variate (SNV), first derivative (FD), second derivative (SD), multiplicative scatter correction (MSC) and smoothing were used for establishing the partial least squares(PLS)models, which was determined the sugar content in navel orange. The results showed that the model developed from 450-1800nm spectroscopy after SNV pre-processing achieved the optimal performance. The correlation coefficient $\left(\mathrm{r}^{2}\right)$ of the calibration set and validation set were 0.9349 and 0.8514 respectively, and the root mean squared error of calibration and validation sets were 0.8017 and 1.1649 , respectively. The research indicated that the Vis-NIR spectroscopy technique was feasible to nondestructive measurement of sugar content in navel orange.
\end{abstract}

Keywords: Vis-NIR spectroscopy, nondestructive measurement, sugar content, navel orange.

\section{Introduction}

Currently, the fresh fruit need to meet certain quality grade requirements before they are shipped to the marketplace. These requirements include fruit outward characteristics (i.e. size, color, and shape) and internal quality attributes (sugar, acid, firmness, etc.). With the continuous improvement of people's living standard, the quality requirements of fruit are more and more focus on internal quality attributes. As a quality attribute of fruits, sugar content will directly impacts on consumers' purchase demand.

Visible-Near infrared spectroscopy (Vis-NIRs) is one of the fastest growing analytical technologies in recent years. It has several advantages compared to traditional chemical methods: speed, non-destructed of sample, avoidance of the use of chemicals and simplicity of sample preparation. Researchers have shown for years that Vis-NIR spectroscopy is a suitable technique for sugar content measurement in various

* Corresponding Author. E-mail: suikelmh@sohu. com 
fruits: nectarine[1], apple[2-5], Nanfeng mandarin[6], and citrus[7]. Vis-NIR was also used for measurement the quality attributes of tea[8], Chlorophyll[9], pork[10], etc. The development of equipment with improved electronic and optical components and the advent of computers capable of effectively processing the information contained in Vis-NIR spectra has facilitated the expansion of this technique in an increasing number of fields.

This paper focuses on the navel orange sugar content results obtained by using different spectral ranges and different spectral pre-processing methods to establish the validation models of partial least squares (PLS).

\section{Materials and Methods}

\subsection{Navel Orange Samples}

Two hundred and seventy navel orange samples were used for the experiment. These navel oranges were harvested from an orchard at Ningdu county, Jiangxi province, China. The samples were placed at room temperature $\left(10^{\circ} \mathrm{C}\right)$ and relative humidity $(60 \%)$ for $168 \mathrm{~h}$ before the experiment. Total of samples were divided into two groups: 185 samples were designed as calibration models set for model development and the remaining as validation set for validation. The selection of samples for calibration and validation was random and the samples' sugar content of validation was among the region of calibration samples' sugar content.

\subsection{Vis-NIR Imaging System}

Figure1 shows the Vis-NIRs imaging system. The system mainly consisted of an imaging spectrograph QualitySpec Pro (Analytical Spectral Devices, Inc., USA) with a

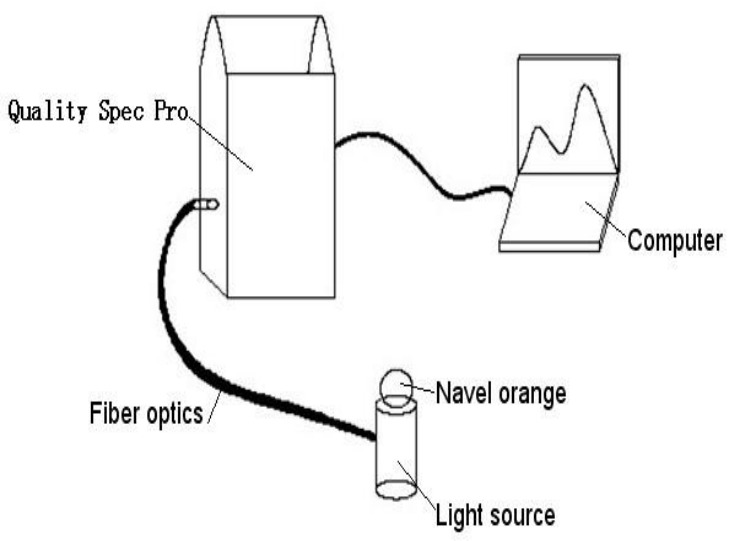

Fig. 1. Vis-NIRs system 
spectral range from $350 \mathrm{~nm}$ to $1800 \mathrm{~nm}$, appropriate lighting sources, optical fiber and a computer. Spectra of the samples were obtained with $1 \mathrm{~nm}$ interval and scan frequency was 10 times. Indico software (version 4.0, Analytical Spectral Devices, Inc., USA) was used for spectra data. Each navel orange was measured 6 times, rotating $60^{\circ}$ was taken on the equatorial zone of the navel orange. The average of the 6 measurements was used later for date processing. In the measurement, note that to prevent light leakage and avoid scratches, scars and other surface defects as much as possible.

\subsection{Experimental Procedure}

After the imaging, Extracted juice of each navel orange flesh was filtered and then taken for sugar content measurement by a digital refractometer (Model PR-101,Atago Co. Ltd, Tokyo, Japan). Each sample was measured 9 times with the squeezing juice. The average of the 9 measurements was used later for date processing.

\subsection{Spectral Data Processing}

The raw Vis-NIR spectral profiles of navel oranges are shown in figure 2. It can be seen that the spectra contain a lot of noises from wavelengths between 350 to $449 \mathrm{~nm}$, and absorption peak due to water around $1000 \mathrm{~nm}$. Thus, the raw spectrum was divided into three different spectral ranges: $450-1000 \mathrm{~nm}, 1000-1800 \mathrm{~nm}$ and $450-1800 \mathrm{~nm}$, and then five different spectral pre-processing methods of standard orthogonal variable transformation (SNV), first derivative (FD), second derivative (SD), multiplicative scatter correction (MSC) and smoothing were used. Spectral pre-processing of raw spectra data were applied to all measured spectra to reduce the effects of high-frequency random noise, baseline drift, sample particle size and light scattering etc, which can effectively adjust near-infrared spectrum[11].

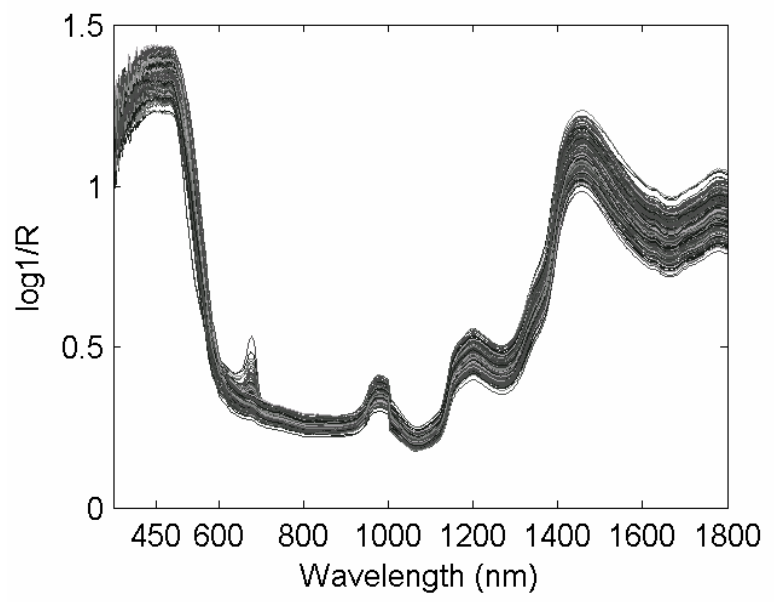

Fig. 2. Raw spectra of navel oranges 
Software Unscrambler (version 9.7, CAMO AS, Trondheim, Norway) and Matlab 7.1(Mathworks, Inc., USA) were used to analysis Vis-NIR spectroscopic data. Spectra from the Indico software were exported to suitable format.

\section{Result and Discussion}

\subsection{Pre-processing Methods}

Partial least squares (PLS) is a very popular regression method in application of Vis-NIR spectroscopy. In this study, PLS regression combined with spectral pre-processing such as derivative, standard normal variate transformation (SNV), multiplicative scatter correction (MSC) and smoothing was used to develop calibration models. PLS analysis was carried out to determine the optimal set of wavelengths and to develop calibration models using the statistical software Unscrambler.

Calibration models were compared using the root mean squared error of calibration (RMSEC), root mean squared error of prediction (RMSEP), and correlation coefficients $\left(\mathrm{r}^{2}\right)$ for calibration and validation. A good model should have a low RMSEC, a low RMSEP and a high $\mathrm{r}^{2}$ value. The calculation of RMSEC indicated how well the model fits the calibration data, and the parameter of RMSEP was reported as an indication of the model validation capability. RMSEC and RMSEP were calculated based on the following formulas:

$$
\begin{gathered}
\text { RMSEC }=\sqrt{\frac{1}{I_{C}-1} \sum_{i=1}^{I_{C}}\left(\hat{y_{i}}-y_{i}\right)^{2}} \\
R M S E P=\sqrt{\frac{1}{I_{P}-1} \sum_{i=1}^{I_{P}}\left(\hat{y_{i}}-y_{i}-\text { bias }\right)^{2}} \\
\text { bias }=\frac{1}{I_{P}} \sum_{i=1}^{I_{P}}\left(\hat{y_{i}}-y_{i}\right)
\end{gathered}
$$

Where: $I_{C^{---}}$number of samples in the calibration set; $I_{P^{---}}$number of samples in the validation set; $y_{i}$----- measured value of the $\mathrm{i}^{\text {th }}$ sample; $\hat{y}_{i}$----- predicted value of the $\mathrm{i}^{\text {th }}$ sample; 


\subsection{Calibration and Validation Models}

Table 1 shows the PLS models results of different pre-processing methods and spectral ranges. The best wavelength range was from 450 to $1800 \mathrm{~nm}$, which was wider than the other two wavelength ranges (450 1000 nm and 1000 1800 nm). It covers the visible and NIR spectral regions. Comparing the results in table 1, it shows that the optimal PLS model is obtained in the range of $450-1800 \mathrm{~nm}$ and by the spectral data preprocessing method of SNV. For calibration, the correlation coefficient $r^{2}$ and the RMSEC is 0.8514 and 0.8017 respectively. For validation, the correlation coefficient $\mathrm{r}^{2}$ and the RMSEP is 0.8514 , and 1.1649 respectively.

Table 1. PLS models results of different pre-processing methods and spectral ranges

\begin{tabular}{lcccccc}
\hline \multirow{2}{*}{$\begin{array}{l}\text { Wavelength } \\
\text { Range/nm }\end{array}$} & $\begin{array}{c}\text { Spectral } \\
\text { Pre-processing } \\
\text { Method }\end{array}$ & Factors & & \multicolumn{2}{c}{ Calibration } & \multicolumn{2}{c}{ Validation } \\
\cline { 3 - 7 } $450 \sim 1800$ & & $\mathrm{r}^{2}$ & RMSEC & $\mathrm{r}^{2}$ & RMSEP \\
& SNV & 17 & 0.9349 & 0.8017 & 0.8514 & 1.1649 \\
& FD & 7 & 0.8556 & 1.1941 & 0.8063 & 1.3302 \\
& SD & 6 & 0.7885 & 1.4452 & 0.6972 & 1.6630 \\
& MSC & 18 & 0.9397 & 0.7718 & 0.8434 & 1.1960 \\
$450 \sim 1000$ & Smoothing & 19 & 0.9315 & 0.8224 & 0.8305 & 1.2443 \\
& SNV & 13 & 0.8875 & 1.0587 & 0.7739 & 1.4372 \\
& FD & 5 & 0.8044 & 1.3900 & 0.7924 & 1.3772 \\
& SD & 6 & 0.7634 & 1.5287 & 0.7056 & 1.6340 \\
& MSC & 14 & 0.9020 & 0.9840 & 0.8000 & 1.3514 \\
& Smoothing & 13 & 0.8894 & 1.0451 & 0.7691 & 1.4524 \\
& SNV & 14 & 0.8857 & 1.0627 & 0.8471 & 1.1819 \\
& FD & 7 & 0.8889 & 1.0475 & 0.7634 & 1.4700 \\
& SD & 5 & 0.8854 & 1.0639 & 0.5272 & 2.0780 \\
& MSC & 14 & 0.8853 & 1.0644 & 0.8475 & 1.1804 \\
& Smoothing & 15 & 0.8728 & 1.1209 & 0.8505 & 1.1684 \\
\hline
\end{tabular}

Figure 3 shows the calibration result using PLS and SNV spectral pre-processing in the wavelength range of $450-1800 \mathrm{~nm}$ for 185 intact navel oranges with a calibration correlation coefficient $\mathrm{r}^{2}$ of 0.8514 , a RMSEC of 0.8017 and figure 4 shows the validation result for the left 85 samples with a validation correlation coefficient $r^{2}$ of 0.8514 , a RMSEP of 1.1649. As the error that happened in chemical test and instruments, the result of validation was not good enough, so the next step research is to change the method to improve the predict capability. It was concluded that Vis-NIR reflection method yields a satisfied estimate of sugar content value in intact navel orange. 


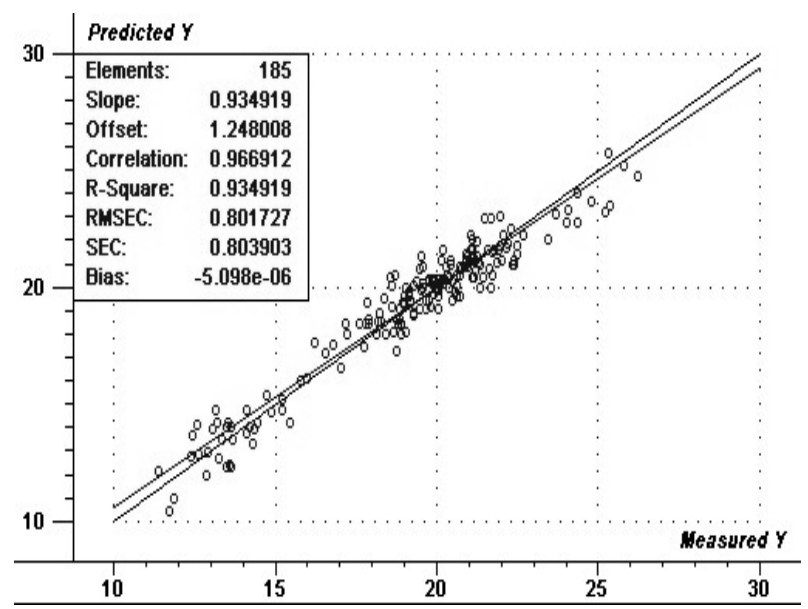

Fig. 3. The optimal model of the calibration set

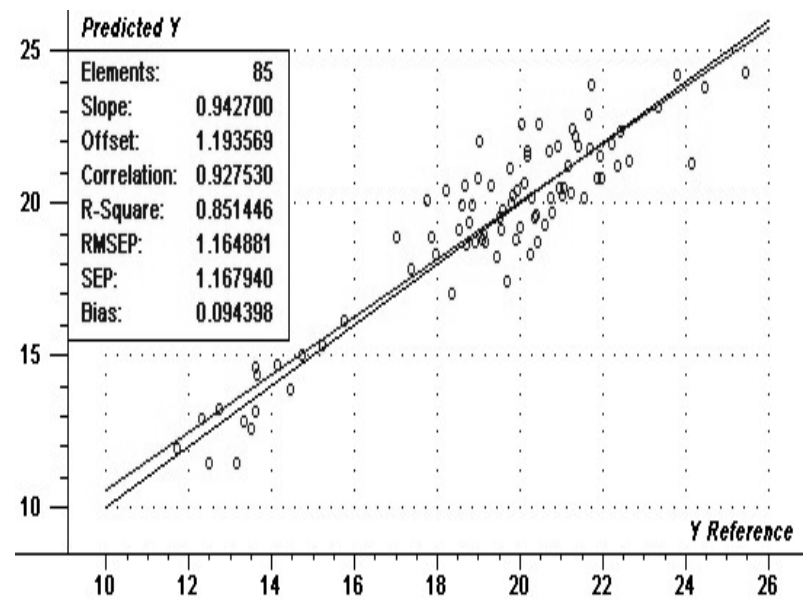

Fig. 4. The optimal model of the validation set

\section{Conclusions}

This research indicated that it is possible to develop a nondestructive method for measuring the navel orange sugar content by Vis-NIR spectroscopy. The results showed that the PLS model developed from 450-1800nm spectroscopy after SNV pre-processing method achieved the optimal performance. The correlation coefficient (r2)of the optimal model of the calibration set and validation set were 0.9349 and 0.8514 respectively and root mean squared error of calibration and root mean squared error of validation were 0.8017 and 1.1649 respectively. It can be concluded that 
VIS/NIR spectroscopy could be a reliable, accurate, and fast method for measurements sugar content of navel orange.

\section{Acknowledgement}

This work was supported in part by the National Natural Science Foundation of China (No. 30760101), Jiangxi Provincial Department of Science and Technology (No. 2009BNB05705) and Jiangxi Provincial Department of Education (No.GGJ08513).

\section{References}

1. Yu, X., Liu, M., Cheng, R.: A Study on Non-destructive Measurement of Nectarine Sugar Content by Means of Spectral Imaging. Acta Agriculturae Universitatis Jiangxiensis 29(6), 1035-1038 (2007)

2. Qing, Z., Ji, B., Shi, B., et al.: Improving Apple Fruit Quality Predictions by Effective Correction of Vis-NIR Laser Diffuse Reflecting Images. Spectroscopy and Spectral Analysis 28(6), 1273-1277 (2008)

3. Zhao, J., Zhang, H., Liu, M.: Non-destructive determination of sugar contents of apples using near infrared diffuse reflectance. Transactions of the CSAE 21(3), 162-165 (2005)

4. Zhao, J., Zhang, H., Liu, M.: Preprocessing Methods of NearInfrared Spectra for Simplifying Prediction Model of Sugar Content of Apples. Acta Optica Sinica 26(1), 136-140 (2006)

5. Zhu, D., Yu, D., Zhang, D.: A Quick and Non-destructive Measurement of Soluble Solids Content of Apple Based on Visible/Near Infrared Spectroscopy. Jinghua College of Vocation and Technology 9(6), 37-41 (2009)

6. Liu, Y., Luo, J., Chen, X.: Analysis of Soluble Solid Content in Nanfeng mandarin fruit with Visible Near infrared spectroscopy. J. Infrared Millim. Waves 27(2), 119-122 (2008)

7. Lu, H., Fu, X., Xie, L., Ying, Y.: Estimation of Soluble Solids Content of Intact Citrus Fruit by Vis/NIR Spectroscopy. Spectroscopy and Spectral Analysis 27(9), 1727-1730 (2007)

8. Zhao, J., Guo, Z., Chen, Q., Lü, Q.: Feasibility Study on Use of Near-Infrared Spectroscopy in Quantitative Analys is of Catechins in Green Tea. Acta Optica Sinica 28(12), 2302-2306 (2008)

9. Li, Q., Huang, Y., Zhang, G., et al.: Chlorophyll Content Nondestructive Measurement Method Based on Vis/NIR Spectroscopy. Spectroscopy and Spectral Analysis 29(12), 3275-3278 (2009)

10. Liu, K., Cheng, F., Lan, H.: Visible/NIR Analysis of Fat, Protein and Water in Chilled Pork. Spectroscopy and Spectral Analysis 29(1), 102-105 (2009)

11. Lu, W., Yuan, H., Xu, G., et al.: Modern Near Infrared Spectroscopy Analytical Technology, pp. 35-37. China Petrochemical Press, Beijing (2007)

12. Peng, Y., Lu, R.: Analysis of spatially resolved hyperspectral scattering images for assessing apple fruit firmness and soluble solids content. Postharvest Biology and Technology 48, 52-62 (2008)

13. Sánchez, N.H., et al.: Robustness of models based on NIR spectra for sugar content prediction in apples. Near Infrared Spectrosc. 11, 97-107 (2003) 